\title{
Major Antigen of Liver Kidney Microsomal Autoantibodies in Idiopathic Autoimmune Hepatitis Is Cytochrome P450db1
}

\author{
Michael P. Manns, ${ }^{\star}$ Eric F. Johnson, ${ }^{\star}$ Keith J. Griffin, ${ }^{\ddagger}$ Eng M. Tan, ${ }^{\star}$ and Kevin F. Sullivan* \\ ${ }^{*} W$. M. Keck Autoimmune Disease Center, and ${ }^{\ddagger}$ Division of Biochemistry, Department of Basic and Clinical Research, \\ Scripps Clinic and Research Foundation, La Jolla, California 92037
}

\begin{abstract}
Type 1, liver kidney microsomal autoantibodies (LKM-1) are associated with a subgroup of idiopathic autoimmune type, chronic active hepatitis (CAH). The antigenic specificity of LKM-1 autoantibodies from 13 patients was investigated by immunoblot analysis of human liver microsomal proteins. Polypeptides of 50, 55, and $64 \mathrm{kD}$ were detected with these antisera. A high titer LKM-1 serum was selected to screen a human liver $\lambda$ gt11 cDNA expression library, resulting in the isolation of several complementary (c)DNA clones. Autoantibodies affinity purified from proteins expressed by two of the immunopositive cDNA clones, HLD8.2 and HLD13.2, specifically react with a $50-\mathrm{kD}$ protein of human liver microsomes and display immunofluorescence staining of the proximal renal tubular epithelia characteristic of LKM-1 sera. Determination of the sequence of HLD8.2 revealed that it encodes a recently described cytochrome P450db1. A bacterial fusion protein constructed from HLD8.2 proved to be a specific and sensitive diagnostic reagent. All sera from patients with LKM-1 positive liver disease react with this fusion protein. No reaction was seen, however, for sera from patients with other types of autoimmune liver diseases, viral hepatitis, systemic immunological disorders, or healthy controls.
\end{abstract}

\section{Introduction}

This study was directed toward the identification of the proteins recognized by type 1 liver kidney microsomal antibodies (LKM-1) ${ }^{1}$ autoantibodies associated with some forms of chronic active hepatitis (CAH). CAH is a clinically and morphologically defined syndrome arising from a variety of differ-

A preliminary report on this work was presented at the Annual Meeting of the American Gastroenterological Association, 14-18 May 1988, New Orleans, LA.

Address reprint requests to Dr. Manns, I. Medizinische Klinik and Poliklinik, Langenbeckstrasse 1, D-6500 Mainz 1, FRG.

Received for publication 7 June 1988 and in revised form 1 November 1988.

1. Abbreviations used in this paper: AMA: antimitochondrial antibodies, CAH: chronic active hepatitis, HBsAg: hepatitis B surface antigen, HBV: hepatitis $B$ virus, LKM: liver kidney microsomal antibodies, PBC: primary biliary cirrhosis, SLA: soluble liver antigen.

J. Clin. Invest.

(c) The American Society for Clinical Investigation, Inc.

0021-9738/89/03/1066/07 \$2.00

Volume 83, March 1989, 1066-1072 ent etiologies $(1,2)$. In addition to hepatitis B virus (HBV) induced $\mathrm{CAH}, \mathrm{HBV}$ negative $\mathrm{CAH}$ consists of a diverse group of diseases, including non-A, non-B viral $\mathrm{CAH}$, and idiopathic autoimmune type $\mathrm{CAH}$. Autoimmune $\mathrm{CAH}$ is often associated with a predominance of female over male patients $(8: 1)$, with hypergammaglobulinemia and nonhepatic autoimmune syndromes, as well as with HLA phenotypes B8 and DR3 (1). Analysis of circulating autoantibodies has defined three subgroups of idiopathic autoimmune type CAH (1-3). LKM-1 autoantibodies characterize one subgroup of idiopathic autoimmune CAH (1-7). LKM-1 antibodies are not associated with either classical autoimmune $\mathrm{CAH}$, which is positive for antinuclear antibody $(1,7,8)$ or with the third subgroup, characterized by autoantibodies against a soluble liver antigen (SLA) (1). LKM autoantibodies were originally described by immunofluorescence exhibiting a typical cytoplasmic staining of hepatocytes and proximal renal tubular epithelia (9). Sera from such patients react on Western blots with a 50-kD microsomal protein $(10,11)$. LKM-1 antibodies are distinguished from LKM-2 antibodies, which have a similar immunofluorescence pattern, but which are specifically associated with drug-induced hepatitis caused by tienilic acid (ticrynafen) (11).

In the present study, we describe the heterologous pattern of LKM-1 autoantibody specificities assayed by immunoblot analysis of human liver microsomal proteins. In order to identify and characterize the antigen(s) recognized by LKM-1 autoantibodies, a high titer LKM-1 positive serum was used as a probe to screen a $\lambda g t 11$ cDNA expression library. We report here the isolation of a human liver cDNA that corresponds to the 50-kD antigen recognized by LKM-1 autoantibodies. Sequence analysis of this cDNA reveals that it encodes cytochrome $\mathrm{P} 450 \mathrm{db} 1$, the cytochrome $\mathrm{P} 450$ responsible for widespread polymorphism in the ability to metabolize certain antihypertensive drugs including debrisoquine (12). A bacterial fusion polypeptide produced in plasmid pATH 11 proved to be a specific and sensitive diagnostic tool for the detection of LKM-1 autoantibodies in patient sera.

\section{Methods}

Patient sera. 13 LKM-1 positive sera from patients with hepatitis B surface antigen ( $\mathrm{HBsAg}$ ) negative chronic hepatitis were the focus of this study. Three sera that exhibited a characteristic LKM type immunofluorescence pattern but from patients with no evidence of liver disease were also examined. These three sera from patients with idiopathic thrombocytopenia, scleroderma, and chronic sinusitis, respectively, were selected on the basis of their LKM immunofluorescence from several hundred autoimmune sera collected at Scripps Clinic and Research Foundation. They are tabulated together with the $13 \mathrm{CAH}$ sera in Table I to emphasize their distinct antibody specificity. 
Table I. Clinical and Immunological Features of Patients with LKM-Antibodies

\begin{tabular}{|c|c|c|c|c|c|c|c|}
\hline \multirow[b]{2}{*}{ Patient } & \multirow[b]{2}{*}{ Age/Sex } & \multirow[b]{2}{*}{ Diagnosis } & \multicolumn{2}{|r|}{ LKM-Ab } & \multicolumn{3}{|c|}{$\begin{array}{l}\text { Western Blot:human } \\
\text { liver microsomes }\end{array}$} \\
\hline & & & IF & $\mathbf{R}(\mathbf{P 4 5 0 \mathrm { db } 1 )}$ & $50 \mathrm{kD}$ & $55 \mathrm{kD}$ & $64 \mathrm{kD}$ \\
\hline 1 & $52 / \mathrm{F}$ & $\mathrm{CPH}$ & + & + & - & - & - \\
\hline 2 & $25 / F$ & $\mathrm{CAH} / \mathrm{Ci}$ & + & + & + & + & - \\
\hline 3 & $64 / F$ & CAH & + & + & - & - & - \\
\hline 4 & $61 / \mathrm{M}$ & $\mathrm{CPH}$ & + & + & - & - & - \\
\hline 5 & $44 / F$ & $\mathrm{CAH}$ & + & + & + & - & - \\
\hline 6 & $37 / F$ & $\mathrm{CAH}$ & + & + & + & - & - \\
\hline 7 & $41 / F$ & $\mathrm{CAH}$ & + & + & - & - & - \\
\hline 8 & $22 / \mathrm{F}$ & $\mathrm{CAH}$ & + & + & - & - & - \\
\hline 9 & $25 / F$ & $\mathrm{CAH}$ & + & + & + & - & + \\
\hline 10 & $14 / F$ & $\mathrm{CAH} / \mathrm{Ci}$ & + & + & + & - & + \\
\hline 11 & $32 / \mathrm{M}$ & CAH & + & + & + & - & + \\
\hline 12 & $24 / F$ & $\mathrm{CAH}$ & + & + & + & - & - \\
\hline 13 & $24 / F$ & CAH & + & + & - & - & + \\
\hline 14 & $52 / \mathrm{M}$ & ITP & + & - & - & - & - \\
\hline 15 & $72 / \mathrm{F}$ & Scleroderma & + & - & - & - & - \\
\hline 16 & $47 / M$ & $\begin{array}{l}\text { Chronic } \\
\text { sinusitis/HLP }\end{array}$ & + & - & - & - & - \\
\hline
\end{tabular}

None of the above patients had HBsAg or autoantibodies to smooth muscle antigen, mitochrondria or soluble liver antigens. Only patient 15 (scleroderma) had antinuclear antibodies by IF. Abbreviations used in this table: LKM-Ab, liver kidney microsomal antibodies; IF, immunofluorescence; $\mathbf{R}(\mathbf{P} 450 \mathrm{db} 1)$, recombinant $\mathrm{P} 450 \mathrm{db} 1$ antigen; $\mathrm{CAH} / \mathrm{Ci}$, chronic active hepatitis/cirrhosis of the liver; $\mathrm{CPH}$, chronic persistent hepatitis; ITP, idiopathic thrombocytopenic purpura; HLP, hyperlipoproteinemia.

In addition, sera from 103 patients with various liver diseases, from 34 patients with systemic immunological disorders, from 20 patients with various malignancies, and from 20 healthy blood donors were included in this study. The group of 103 patients with liver diseases consisted of patients with classical autoimmune type "lupoid" CAH ( $n$ $=10$ ) positive for antinuclear antibodies, patients with SLA antibody positive autoimmune type CAH $(n=10)$, hepatitis B virus-induced CAH $(n=10)$, CAH non-A-non-B $(n=10)$, CAH due to hepatitis D (delta) virus infection $(n=10)$, acute viral hepatitis $\mathrm{A}(n=5), \mathrm{B}(n$ $=10$ ), non-A-non-B $(n=6)$, antimitochondrial antibody (AMA) positive primary biliary cirrhosis $(\mathrm{PBC})(n=20)$, and extrahepatic cholestasis due to bile duct calculi $(n=20)$. The 34 patients with systemic immunological disorders consisted of patients with systemic lupus erythematosus $(n=11)$, scleroderma $(n=5)$, rheumatoid arthritis $(n=4)$, Wegener's granulomatosis $(n=1)$, Reiter's disease $(n=4)$, Crohn's disease $(n=6)$, sarcoidosis $(n=3)$. The group of 20 patients with malignancies consisted of carcinoma of the stomach $(n=3)$, pancreas $(n=2)$, colon $(n=5)$, and rectum $(n=4)$, malignant melanoma $(n=3)$, as well as lung $(n=2)$ and breast cancer $(n=1)$. All these sera were tested for LKM antibodies by indirect immunofluorescence on rodent liver and kidney sections, Western blots with human liver microsomes and against recombinant LKM-1 antigen as will be described below. These sera were collected at the I. Department of Internal Medicine, University of Mainz, FRG, which is a reference laboratory for liver diseases.

Immunological methods. Autoantibodies were tested by immunofluorescence on mouse liver and kidney cryostat tissue sections $(6,9)$. LKM and SLA antibodies were assayed by radioimmunoassay as described previously $(1,6)$. Immunoblotting analysis of patient sera was performed as described previously (13). Human and rabbit liver microsomes (14) and bacterial lysates containing fusion protein were used as antigens in immunoblot analyses. ${ }^{125} \mathrm{I}$-protein $\mathrm{A}$ (ICN) diluted to $200,000 \mathrm{cpm} / \mathrm{ml}$ in $3 \%$ milk powder PBS/Tween 20 was used to detect human IgG bound to antigens attached to nitrocellulose filters.

Isolation and characterization of $c D N A$ clones. A human liver cDNA library constructed in $\lambda$ gt 11 was obtained commercially (Clontech, Palo Alto, CA). Phage were plated, and protein expression was induced with IPTG saturated nitrocellulose filters in duplicate as described by Young and Davis (15). Filters were processed for immunodetection using a high titer LKM-1 autoantibody positive serum. Following plaque purification, phage were grown in Escherichia coli LE-392 for DNA isolation as described by Maniatis et al. (16). After restriction digestion with Eco RI, cDNA inserts were subcloned into M13MP18 or Bluescript (Stratagene Laboratories, La Jolla, CA) for further analysis. DNA sequence analysis was performed using the dideoxy method (17). The sequence of the cDNA was determined on both strands from a set of overlapping restriction fragments subcloned into M13 or Bluescript.

Preparation of affinity purified antibodies. Fusion proteins expressed by two LKM-1 positive cDNA clones or an unrelated cDNA were absorbed to nitrocellulose filters. The filters were then incubated with 1:200 dilution of patient serum in 3\% milk powder PBS/Tween 20 for $60 \mathrm{~min}$ at room temperature and then washed extensively in PBS/

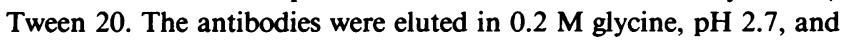
immediately neutralized by addition of $1 \mathrm{M}$ Tris buffer, $\mathrm{pH} 8$. The antibody was concentrated 25 -fold for use in immunoblotting and immunofluorescence experiments.

Fusion protein analysis. For immunoblot analysis using the cloned antigen, the cDNA was subcloned into the fusion protein vector, pATH 11 (kindly provided by T. J. Koerner, Duke University, Durham, NC). pATH 11 contains the $5^{\prime}$ portion of the $E$. coli trpE gene, encoding $36 \mathrm{kD}$ of anthranilate synthetase followed by a polylinker that possesses an Eco RI site in the same translational frame as the Eco $\mathrm{RI}$ site of $\lambda \mathrm{gt} 11$. After ligation and transformation into $E$. coli $\mathrm{RR}-1$, colonies were selected, cultured, and processed for immunoblot analysis as described previously (18).

\section{Results}

Immunoblotting analysis. Sera from 13 hepatitis patients, each positive for LKM-1 antibodies as determined by immunofluorescence were tested for reactivity with human microsomal proteins by immunoblot analysis (Fig. 1). These patients were diagnosed as having $\mathrm{HBsAg}$ negative chronic active or persistent hepatitis. Among the LKM-1 positive CAH sera, a heterogeneous pattern of reactivity with human microsomal proteins was observed. Seven of the LKM-1 positive hepatitis sera showed reactivity with a 50-kD microsomal protein of human liver microsomes. Sera 5 and 6 showed only very weak reactivity. This is consistent with previous reports $(10)$ of the reactivity of LKM-1 antibodies with a $50-\mathrm{kD}$ protein in human liver microsomes. Four sera reacted with a different microsomal protein of $64 \mathrm{kD}$ (Fig. 1, sera 9, 10, 11, 13) and one with an additional protein at $55 \mathrm{kD}$ (Fig. 1, serum 2).

Three sera from patients with idiopathic thrombocytopenia, scleroderma, or chronic sinusitis, respectively, were also tested because they exhibited immunofluorescence on sections of mouse liver and kidney similar to that of LKM-1 autoantibodies $(6,9)$. These patients did not exhibit evidence of liver disease, and the three sera did not react with human microsomal proteins as judged by immunoblotting (Fig. 1). Additional sera from 103 patients with degenerative liver diseases including autoimmune CAH with associated SLA and ANA autoantibodies, viral hepatitis, primary biliary cirrhosis, and extrahepatic cholestasis due to bile duct calculi were negative. Sera from 34 patients with systemic immunologic disorders, 
from 20 patients with various malignancies, as well as from 20 healthy blood donors were also negative by immunoblot analysis.

Isolation of an LKM-1 cDNA. A high titer LKM-1 autoantibody positive serum was selected to screen a human liver cDNA expression library constructed in $\lambda g t 11$. This serum (Fig. 1, serum 10) reacts with both the 50- and 64-kD microsomal protein on Western blots and exhibited the typical LKM-1 immunofluorescence pattern on mouse tissue. From a screen of $2 \times 10^{6}$ phage several positive signals were identified and 5 phage were plaque purified. Restriction analysis of DNA purified from each phage revealed that each contained a 1,200 bp cDNA insert. Two of these clones, HLD8.2 and HLD13.2, were selected for further characterization.

Immunological identification of $c D N A$ encoded antigen. To determine the identity of the antibodies reactive with the cDNA encoded antigen, purified phage from either HLD8.2, HLD13.2 or an unrelated cDNA, GTE-411 (18), were plated on $E$. coli Y1090, and antigen production was induced with IPTG saturated nitrocellulose filters. The filters, bearing cDNA encoded, fusion protein antigens, were incubated with a 1:200 dilution of LKM-1 serum 10. After extensive washing, bound antibodies were eluted from the filters and used for immunoblot analysis of human microsomal proteins. Antibodies eluted from proteins expressed by each of the LKM-1 positive cDNA clones reacted specifically with the $50-\mathrm{kD}$ microsomal protein of human liver microsomes (Fig. $2 a$, lanes 2 and 3). The specificity of the immunoaffinity purification procedure was verified by the lack of reactivity of antibodies recovered from control filters (Fig. $2 a$, lane 4 ) and by the lack of reactivity of the purified antibodies with the $64-\mathrm{kD}$ antigen, recognized by the original serum. Furthermore, using the immunofluorescence assay, autoantibodies affinity purified from proteins expressed by cloned LKM-1 cDNAs reacted with the proximal renal tubules of mouse with a pattern indistinguishable from that of the original serum (Fig. $2 b$ ). These experiments demonstrate that clones HLD8.2 and HLD13.2 express an epitope or epitopes in common with the 50-kD human microsomal antigen recognized by LKM-1 autoantibodies.

Sequence of HLD8.2: a human class IID cytochrome P450. The nucleotide sequences of the termini of HLD8.2 and HLD13.2 were identical. Therefore, only the cDNA HLD8.2 was used for further analysis. The complete 1,205 -bp sequence of HLD8.2 (Fig. 3) was determined using the dideoxy method (17) from a set of overlapping restriction fragments subcloned in M13MP18 or Bluescript. A single long open reading frame encoding $\sim 42 \mathrm{kD}$ of polypeptide is evident extending for $1,120 \mathrm{bp}$, followed by a consensus polyadenylation site, AATAAA. Extensive sequence identity between HLD8.2 and two rat cDNAs encoding class IID cytochrome P450s (19) indicated that HLD8.2 could encode a human homologue of these enzymes. This was confirmed by the recent publication of the sequence of the human class IID cytochrome P450 (P450db1) (12). An insertion of 9 bp relative to the sequence reported by Gonzalez et al. (12) is observed at position 866 of HLD8.2. This sequence was also found in an independently isolated cDNA from a different individual corresponding to a b-type variant as described by Gonzalez et al. (12) and is homologous to the same region in both rat IID cDNAs (19). In addition, four single-base substitutions were observed within the coding region leading to two differences in the predicted

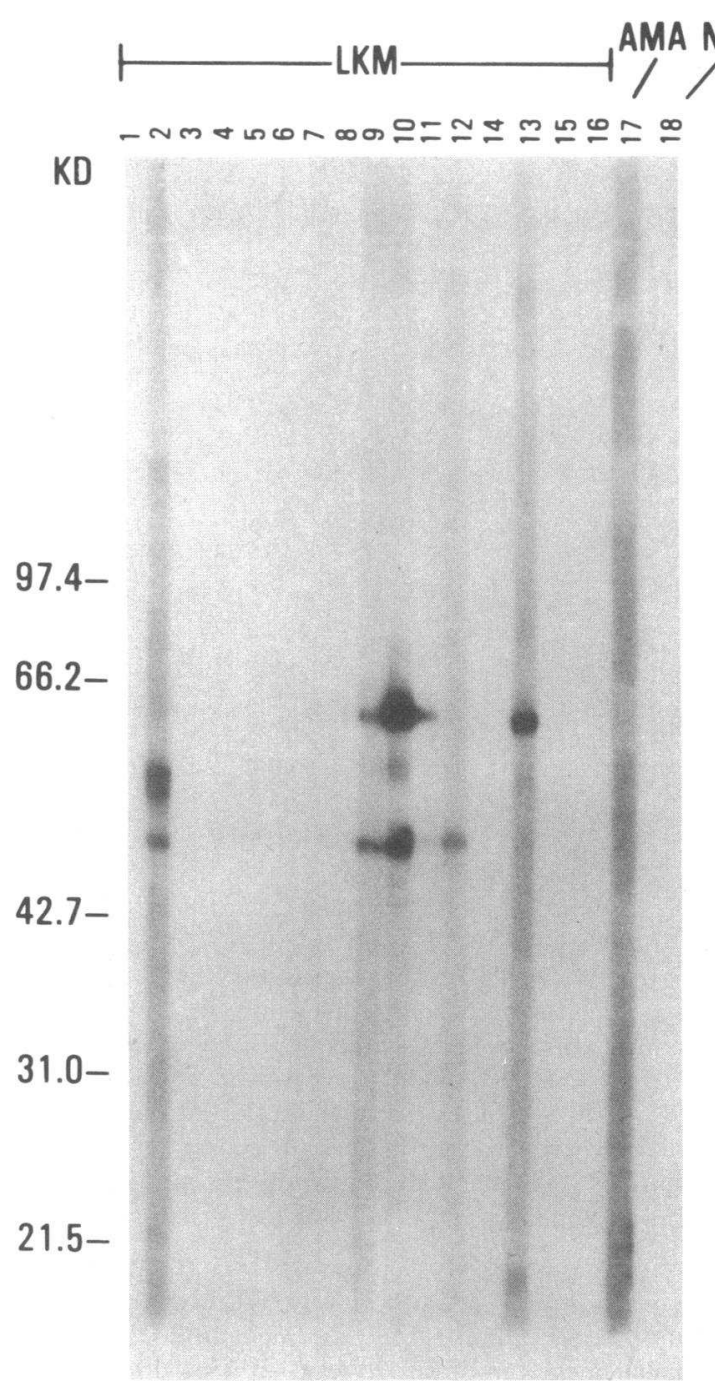

\section{AG: Human Liver Microsomes}

Figure 1. Immunoblot analysis of LKM-1 positive sera. Human liver microsomal proteins were resolved by electrophoresis and transferred to nitrocellulose (13). Strips were cut from the filter in widths corresponding to $\sim 12 \mu \mathrm{g}$ protein per strip, and each strip was incubated with the human serum $(1: 100)$ identified at the top of each lane. Antibody binding was detected with ${ }^{125}$ I-protein A. Lanes 1-13, LKM-1 positive hepatic disease sera; lanes 14-16, sera from nonhepatic disease patients exhibiting LKM-type immunofluorescence; lane 17 , serum from a primary biliary cirrhosis patient with antimitochondrial antibodies (AMA); and lane 18, normal human serum (NHS). The reactivities of sera 5 and 6 with the 50-kD antigen were weak and were not reproduced in the photograph.

amino acid sequences (Fig. 3). Human cytochrome P450 IID is a microsomal protein with an apparent $M_{\mathrm{r}}$ of $50,000(20)$, which corresponds in size to the $50-\mathrm{kD}$ antigen identified in human liver microsomes by the affinity purified autoantibody.

The use of recombinant antigen for detection of LKM-I autoantibodies in patient sera. Because the clinical distinction between non-A, non-B viral, and autoimmune hepatitis has important implications for disease management, it was of interest to examine the utility of the cloned LKM-1 antigen as an assay for LKM-1 autoantibodies. The HLD8.2 cDNA was subcloned into pATH11, an $E$. coli trpE based fusion protein 

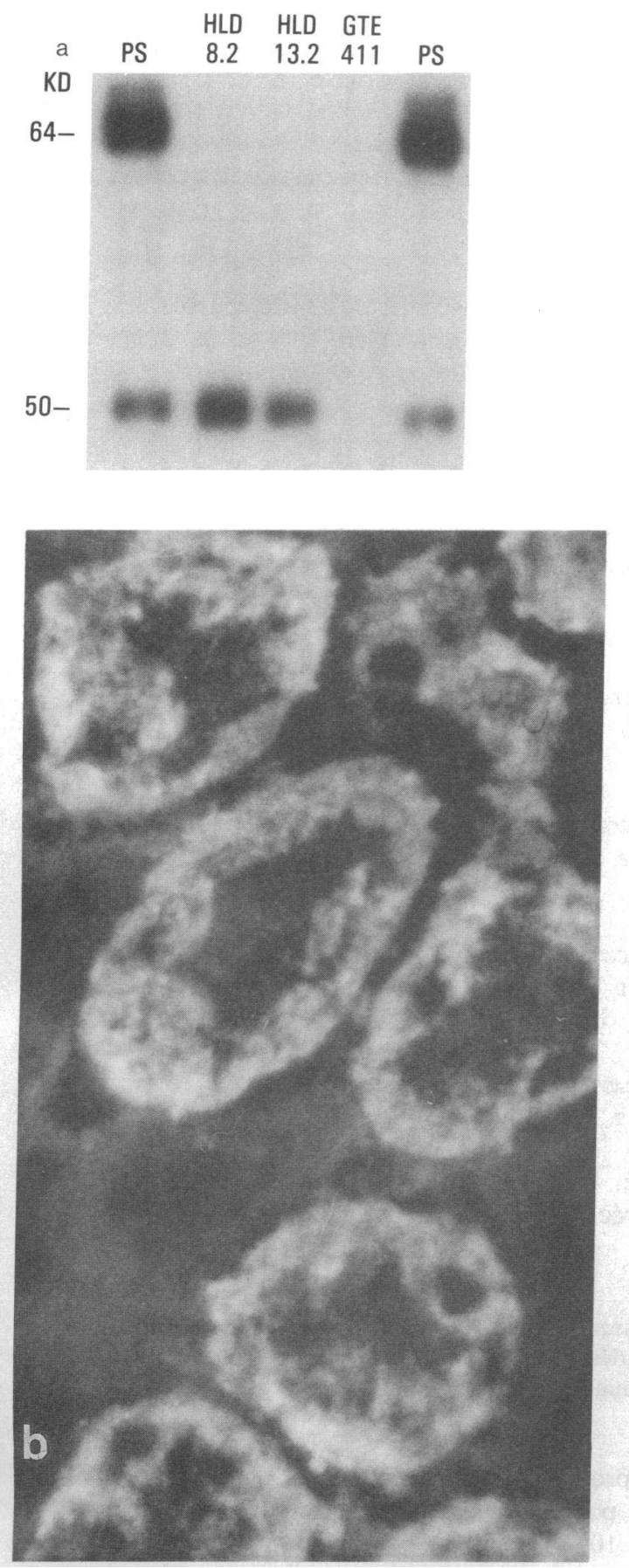

Figure 2. The 50-kD antigen is encoded by HLD8.2 and HLD13.2. Antibodies were affinity purified from nitrocellulose filters bearing cDNA encoded antigens as described in Methods. $(A)$ Immunoblot of human liver microsomes (12 $\mu \mathrm{g}$ protein) probed with whole LKM-1 antibody positive patient serum 10 (PS) at a 1:800 dilution or with affinity purified autoantibodies isolated from $\lambda g t 11$ clones HLD8.2, HLD13.2 or a control cDNA, GTE411. (B) Cytoplasmic immunofluorescence staining of mouse proximal renal tubular cells with autoantibodies affinity purified from antigen encoded by $\lambda g t 11$ clone HLD8.2 ( $\times 50)$.

vector possessing an Eco RI site in the same translational frame as $\lambda g t 11$. Western blot analysis of bacterial lysates probed with the LKM-1 positive serum used to screen the cDNA library showed reactivity with a $72-\mathrm{kD}$ fusion protein, which represents a $36-\mathrm{kD}$ portion of anthranilate synthetase (trp E) and the 42-kD polypeptide encoded by the cDNA when placed in the correct orientation. No reaction was observed with lysates from bacteria bearing the pATH 11 cDNA construct in the opposite orientation or with vector alone (Fig. 4). The reactivity of all of the antisera toward the bacterial lysates was then examined by immunoblotting. Only sera showing the LKM-1 fluorescence pattern that were derived from patients with liver disease (Fig. 5, lanes 1-13) (Table I) reacted with the fusion protein. This included those LKM-1 sera that were negative in immunoblots with human liver microsomes (compare Figs. 1 and 5). In contrast, the three sera displaying LKM-1 type immunofluorescence from individuals with nonhepatic immunological disorders and without clinically apparent liver disease failed to react with the cloned antigen (Fig. 5, lanes 14-16; Table I, patients 14-16), as did sera from 155 patients with various other forms of liver diseases, nonhepatic systemic immunological disorders, malignancies, and from healthy controls. Some of these control sera that were positive for other autoantibody specificities are shown in Fig. 5, lanes 17-20.

\section{Discussion}

The sequence of HLD8.2 demonstrates that it encodes the human class IID cytochrome P450, P450db1 (12). Identification of cytochrome P450db1 as a major autoantigen in LKM-1 positive nonviral CAH rests on three lines of evidence. First, the cDNA encoded antigen is recognized by LKM-1 antisera and specifically selects antibodies from patient sera that are reactive against a $50-\mathrm{kD}$ antigen in human liver microsomes. The molecular weight and subcellular localization of cytochrome P450db1 (20) are consistent with this identification. Secondly, all 13 available LKM-1 antibody positive sera from patients with liver disease react with a recombinant fusion protein constructed from the P450db1 cDNA. Neither sera from patients with other forms of autoimmune liver disease, viral hepatitis, nor sera from patients with various systemic immunological disorders, malignancies, or healthy controls react with the recombinant antigen. Thirdly, LKM-1 autoantibodies affinity purified on recombinant antigen reacted with the proximal tubular cells of mouse kidney with an immunofluorescent pattern characteristic of LKM-1 antisera. We conclude that autoantibodies directed against epitopes present on cytochrome P450db1 are a characteristic feature of the LKM-1 subgroup of idiopathic autoimmune hepatitis ${ }^{2}(1,3)$.

The mechanisms triggering the production of LKM-1 autoantibodies to $\mathrm{P} 450 \mathrm{db} 1$ and its relation to the pathogenesis of liver tissue injury remain unknown. LKM-1 autoantibody production could be a secondary phenomenon related to the release of the $P 450 \mathrm{db} 1$ antigen as result of liver damage in individuals genetically able to mount an immune response to it. However, the fact that LKM-1 antibodies were not observed

2. While this manuscript was in review, Zanger et al. (24) provided us with a preprint of their manuscript describing the capacity of four LKM-1 antisera from French children to inhibit the catalytic activity of P450db1. Another report was published (25) describing the reactivity of LKM-1 antisera derived from French children toward rat liver cDNAs. It is thought that these cDNAs code for the rat equivalent of P450dbl based on restriction mapping. 
GGGCCCGCGTGGCGCGAGCAGAGGCGCTTCTCCGTCTCCACCTTGCGCAACTTGGGCCTGGGCAAGAAGTCGTTGGAGCAGTGGGTGACCGAGGAGGCCGCCTGCCTTTGTGCCGCCTTC 120

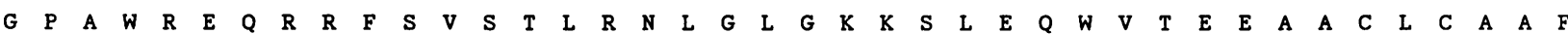
GCCAACCACTCCGGACGCCCCTTTCGCCCCAACGGTCTCTTGGACAAAGCCGTGAGCAACGTGATCGCCTCCCTCACCTGCGGGCGCCGCTTCGAGTACGACGACCCTCGCTTCCTCAGG 240

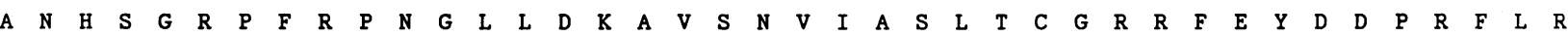

CTGCTGGACCTAGCTCAGGAGGGACTGAAGGAGGAGTCGGGCTTTCTGCGCGAGGTGCTGAATGCTGTCCCCGTCCTCCTGCATATCCCAGCGCTGGCTGGCAAGGTCCTACGCTTCCAA 360

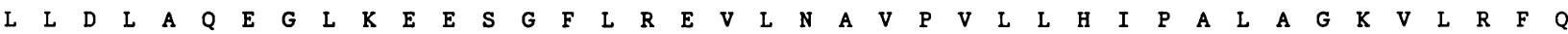
AAGGCTTTCCTGACCCAGCTGGATGAGCTGCTAACTGAGCACAGGATGACCTGGGACCCAGCCCAGCCCCCCCGAGACCTGACTGAGGCCTTCCTGGCAGAGATGGAGAAGGCCAAGGG 480

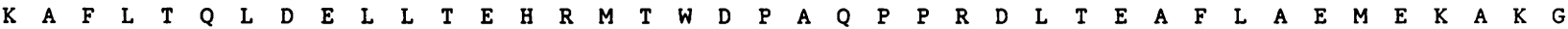
AACCCTGAGAGCAGCTTCAATGATGAGAACCTGCGCATAGTGGTGGCTGACCTGTTCTCTGCCGGGATGGTGACCACCTCGACCACGCTGGCCTGGGGCCTCCTGCTCATGATCCTACAT 600

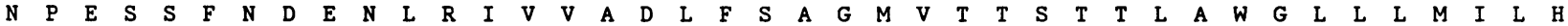
CCGGATGTGCAGCGCCGTGTCCAACAGGAGATCGACGACGTGATAGGGCAGGTGCGGCGACCAGAGATGGGTGACCAGGCTCACATGCCCTACACCACTGCCGTGATTCATGAGGTGCAG 720

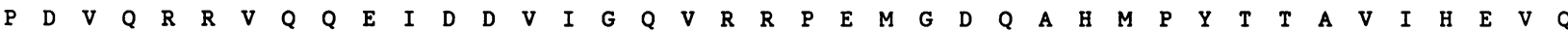

A . CGCTTTGGGGACATCGTCCCCCTGGGTGTGACCCATATGACATCCCGTGACATCGAAGTACAGGGCTTCCGCATCCCTAAGGGAACGACACTCATCACCAACCTGTCATCGGTGCTGAAG 840

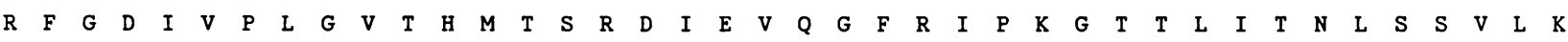
M

GATGAGGCCGTCTGGGAGAAGCCCTTCCGCTTCCACCCCGAACACTTCCTGGATGCCCAGGGCCACTTTGTGAAGCCGGAGGCCTTCCTGCCTTTCTCAGCAGGCCGCCGTGCATGCCTC 960

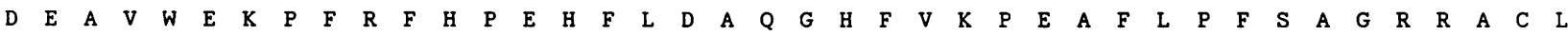
$\mathrm{Y} * * *$

GGGGAGCCCCTGGCCCGCATGGAGCTCTTCCTCTTCTTCACCTCCCTGCTGCAGCACTTCAGCTTCTCGGTGCCCACTGGACAGCCCCGGCCCAGCCACCATGGTGTCTTTGCTTTCCTG 1080

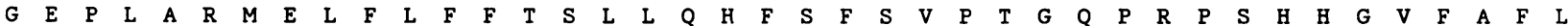

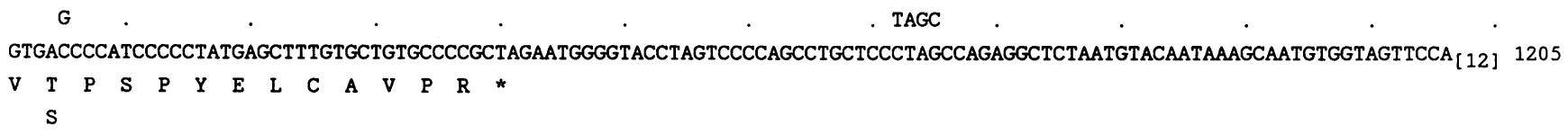

Figure 3. Nucleotide and predicted amino acid sequence of HLD8.2. The nucleotide sequence of cytochrome P450dbl (12) is shown above that of HLD8.2 where they differ. The gap in the sequence of cytochrome P450db1 is denoted by asterisks. The predicted amino acid sequence is displayed below the nucleotide sequence of HLD8.2. Differences in the predicted amino acid sequences are displayed on the fourth line.

for patients with viral hepatitis or other degenerative liver diseases argues against this hypothesis.

One possible mechanism for the involvement of P450db1 in autoimmune disease is that a genetically or environmentally altered form of this P450 may be presented to the immune system in such a way as to induce an immune response. This hypothesis was suggested by the finding that autoantibodies arising in tienilic acid (trycrinafen) induced hepatitis are directed against another human cytochrome P450, P450-8 (11), or P450 $\mathrm{mp} \mathrm{(21)} \mathrm{that} \mathrm{normally} \mathrm{hydroxylates} \mathrm{the} \mathrm{drug.} \mathrm{This}$ lead to speculation that covalent binding of a product of this reaction to the protein might make this $\mathbf{P} 450$ antigenic.

The genetic loci coding for both P450 mp (Cyp 2C9) and P450db1 (Cyp 2D1) are polymorphic. It is also tempting to speculate that an aberrant polypeptide, produced through a missense mutation or by translation of an aberrantly spliced mRNA, could become immunogenic through altered intracellular routing, accumulation, or altered catalytic activity of the resultant protein product. It is estimated that $42-45 \%$ of the caucasian population carry defective alleles of the P450db1 gene with $10 \%$ of the British population showing a profound deficiency in the expression of the enzyme (22). Gonzalez et al. (12) have reported that several individuals fail to accumulate the $\mathrm{P} 450 \mathrm{db} 1$ protein as a result of improper processing of mRNA transcripts derived from at least two different mutant alleles of the P450db1 gene. The identification of cytochrome P450db1 as the antigen recognized by LKM-1 autoantibodies will now enable us to design molecular probes for analysis of the structure and expression of the P450db1 locus in human autoimmune $\mathrm{CAH}$ patients.

Although cytochrome P450db1 was identified in this study as a common autoantigen for LKM-1 positive patient sera, some sera recognized additional proteins. $\mathrm{A} 64-\mathrm{kD}$ protein was detected by 4 of the 13 sera, and one of these sera also recognized a 55-kD protein. The reaction of LKM-1 sera with these additional proteins has not been described previously and indi- 


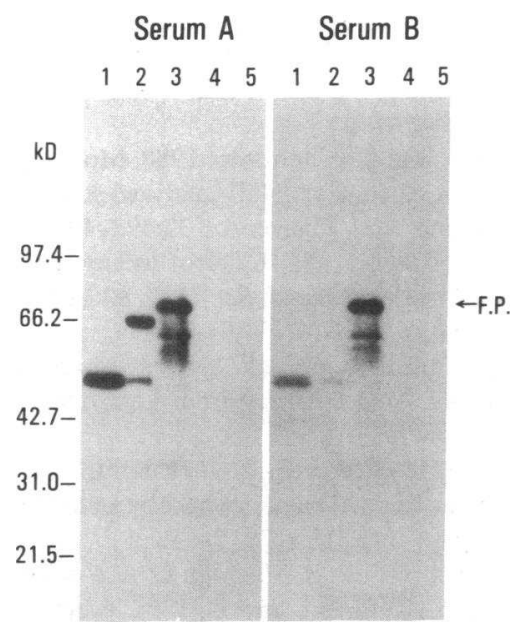

Figure 4. LKM-1 autoantibodies react with pATH 11:HLD8.2 recombinant antigen. cDNA HLD8. 2 was inserted into the $E$. coli trpE-based fusion protein vector, pATH11, and transformed into $E$. coli RR-1. Crude bacterial lysates were electrophoresed in parallel with microsomal protein samples, transferred to nitrocellulose and then probed with two different LKM-1 positive hepatic disease sera. Lane 1, sera with

rabbit liver microsomal proteins $(10 \mu \mathrm{g})$; lane 2 , human liver microsomal proteins (14 $\mu \mathrm{g})$; lane 3, lysate of pATH11:HLD8.2 strain bearing the cDNA insert in the correct orientation; lane 4, lysate of pATH11:HLD8.2 strain bearing the cDNA insert in the incorrect orientation; lane 5 , lysate of $\mathrm{pATH} 11$ vector strain alone. Two sera are shown: serum $A$ reacts with both 50 and $64 \mathrm{kD}$ microsomal antigens while serum $B$ only reacts with the $50 \mathrm{kD}$ microsomal antigen. The fusion polypeptide (FP) has an apparent molecular weight of 72 kD.

cates a heterogeneity in the humoral immune response to liver microsomal antigens among these patients. The affinity purified antibody did not, however, react with either the 64-kD microsomal antigen nor with the $55-\mathrm{kD}$ protein recognized by complete serum 2 (Fig. 1). Therefore the $64-$ and $55-\mathrm{kD}$ epitopes are not encoded by our cloned cDNA. Whether and how antibodies to these additional proteins are related to autoimmune CAH is unknown and has not been addressed in this study. It would be premature to draw any conclusions at this time regarding the role of these autoantibodies in disease pathogenesis.

The recombinant antigen developed in this study provides a reagent for the identification of LKM-1 autoantibodies that are associated with idiopathic autoimmune CAH. The use of the recombinant antigen appears to provide greater sensitivity for the detection of LKM-1 autoantibodies than human liver microsomes as judged by a comparison of Figs. 1 and 5. Autoimmune $\mathrm{CAH}$ represents a significant fraction of $\mathrm{HBs} \mathrm{Ag}$ negative chronic hepatitis (1-3). Immunosuppressive therapy has been shown to improve life expectancy for patients suffering from autoimmune CAH (23). Thus, the identification of LKM-1 positive liver disease would help to select patients who might respond to immunosuppressive therapy. LKM-1 positive liver disease is presently recognized to comprise $10 \%$ of all HBsAg negative $\mathrm{CAH}$ patients and $\sim 25 \%$ of all autoimmune CAH patients (6). The frequency of occurrence in the French population was reported as 5:1,000,000 (3). Improved diagnostic procedures facilitated by the use of the cloned, recombinant antigen could lead to an upward revision of these estimates.

The availability of recombinant LKM-1 antigen will also facilitate investigation of the precise $B$ and $T$ cell immune responses in this autoimmune liver disease. A determination of B cell epitope sequences is in progress. Further studies will be directed toward determination of whether cytochrome

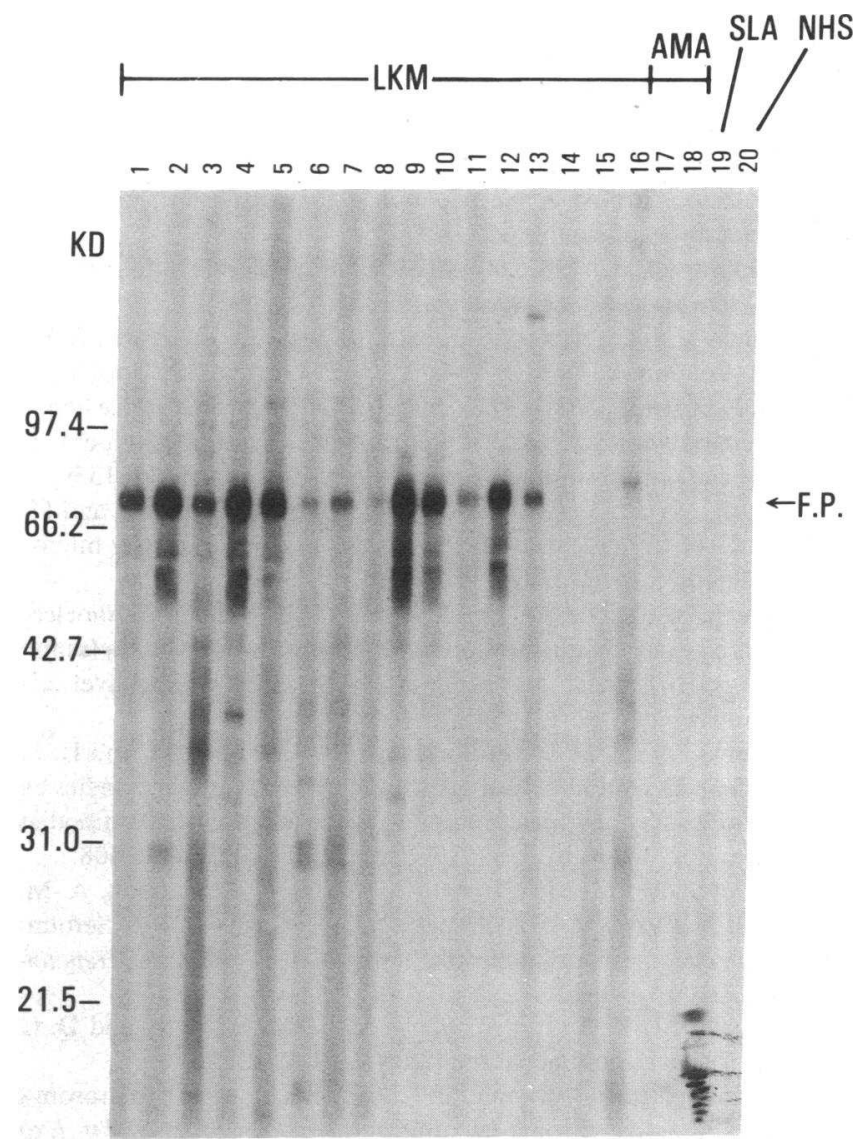

AG: Fusion Protein from Bacterial Lysates

Figure 5. Reactivity of the pATH11:HLD8.2 recombinant antigen with different human sera. Crude bacterial lysates were resolved by electrophoresis and transferred to nitrocellulose filters for immunoblot analysis. Lanes 1-13, LKM-1 positive hepatic disease sera; lanes 14-16, sera from patients without clinically apparent liver disease exhibiting LKM-type immunofluorescence; lanes 17 and 18 , sera from primary biliary cirrhosis patients bearing AMA; lane 19, serum from hepatic disease patient bearing antibodies against a soluble liver antigen (SLA); lane 20, normal human serum.

P450db1 is a target for peripheral T lymphocytes and/or T lymphocytes derived from the inflammatory liver infiltrate. Clarification of the etiology of autoimmune hepatitis may have significant implications for the management of hepatic disease as well as for understanding this and other autoimmune disorders in man.

\section{Acknowledgments}

The authors want to thank doctors F. V. Chisari and K.-H. Meyer zum Buschenfelde for their support, Robert Tukey for the donation of a human liver cDNA library, and T. J. Koerner for the pATH 11 vector. Many thanks go to B. Gandy for help in sequence analysis and C. Peebles for immunofluorescence studies.

Dr. Manns was supported by a fellowship award from the Deutsche Gesellschaft für Verdauungs- und Stoffwechselkrankheiten sponsored by Asche AG during a sabbatical leave from the University of Mainz, West Germany. K. F. Sullivan is a Hulda Irene Dugan Investigator of the Arthritis Foundation. This work was supported in part by U. S. Public Health Service grants AR-32063 (E. M. Tan), GM-39068 (K. F. Sullivan), and GM-31001 (E. F. Johnson). This paper is dedicated to the 60th birthday of K.-H. Meyer zum Buschenfelde. 


\section{References}

1. Manns, M., G. Gerken, A. Kyriatsoulis, M. Staritz, and K.-H. M. Z. Buschenfelde. 1987. Characterisation of a new subgroup of autoimmune chronic active hepatitis by autoantibodies against a soluble liver antigen. Lancet. i:292-294.

2. Maddrey, W. C. 1987. Subdivisions of idiopathic autoimmune chronic active hepatitis. Hepatology. 7:1372-1375.

3. Homberg, J.-C., N. Abuaf, O. Bernard, S. Islam, F. Alvarez, S. H. Khalil, R. Poupon, F. Darnis, V.-G. Levy, P. Grippon, P. Opolon, J. Bernuau, J.-P. Benhamou, and D. Alagille. 1987. Chronic active hepatitis associated with antiliver/kidney microsome antibody type 1: a second type of "autoimmune" hepatitis. Hepatology. 7:1333-1339.

4. Smith, M. G. M., R. Williams, G. Walker, M. Rizzetto, and D. Doniach. 1974. Hepatic disorders associated with liver/kidney microsomal antibodies. Br. Med. J. 2:80-84.

5. Storch, W., L. Cossel, and R. Dargel. 1977. The immunoelectron-microscopical demonstration of antibodies against endoplasmic reticulum (microsomes) in chronic aggressive hepatitis and liver cirrhosis. Immunology. 32:941-945.

6. Manns, M., K.-H. M. Z. Buschenfelde, J. Slusarczyk, and H. P. Dienes. 1984. Detection of liver-kidney microsomal autoantibodies by radioimmunoassay and their relation to anti-mitochondrial antibodies in inflammatory liver diseases. Clin. Exp. Immunol. 57:600-608.

7. Odievre, M., G. Maggiore, J.-C. Homberg, F. Saadoun, A.-M. Courouce, J. Yvart, M. Hadchouel, and D. Alagille. 1983. Seroimmunologic classification of chronic hepatitis in 57 children. Hepatology. 3:407-409.

8. Mackay, I. R., L. I. Taft, M. D. Melb, M. B. Melb, and D. C. Cowling. 1956. Lupoid hepatitis. Lancet. ii:1323-1326.

9. Rizzetto, M., G. Swana, and D. Doniach. 1973. Microsomal antibodies in active chronic hepatitis and other disorders. Clin. Exp. Immunol. 15:331-344.

10. Alvarez, F., O. Bernard, J.-C. Homberg, and G. Kreibich. 1985. Anti-liver-kidney microsome antibody recognizes a 50,000 molecular weight protein of the endoplasmic reticulum. J. Exp. Med. 161:12311236.

11. Beaune, P., P. M. Dansette, D. Mansuy, L. Kiffel, M. Finck, C. Amar, J. P. Leroux, and J. C. Homberg. 1987. Human anti-endoplasmic reticulum autoantibodies appearing in a drug-induced hepatitis are directed against a human liver cytochrome $\mathrm{P}-450$ that hydroxylates the drug. Proc. Natl. Acad. Sci. USA. 84:551-555.

12. Gonzalez, F. J., R. C. Skoda, S. Kimura, M. Umeno, U. M. Zanger, D. W. Nebert, H. V. Gelboin, J. P. Hardwick, and U. A. Meyer, 1988. Characterization of the common genetic defect in humans deficient in debrisoquine metabolism. Nature (Lond.). 331:442-446.
13. Chan, E. K. L., and E. M. Tan. 1987. Human autoantibodyreactive epitopes of SS-B/La are highly conserved in comparison with epitopes recognized by murine monoclonal antibodies. J. Exp. Med. 166:1627-1640.

14. Schwab, G. E., J. L. Raucy, and E. F. Johnson. 1988. Modulation of rabbit and human hepatic cytochrome P450-catalyzed steroid hydroxylations by $\alpha$-naphthoflavone. Mol. Pharmacol. 33:493-499.

15. Young, R. A., and R. W. Davis. 1983. Efficient isolation of genes by using antibody probes. Proc. Natl. Acad. Sci. USA. 80:11941198.

16. Maniatis, T., E. F. Fritsch, and J. Sambrook. 1982. In Molecular Cloning. A Laboratory Manual. Cold Spring Harbor Laboratory, Cold Spring Harbor, NY. pp. 545.

17. Sanger, F., A. R. Coulson, B. G. Barrell, A. J. H. Smith, and B. A. Roe. 1980. Cloning in single-stranded bacteriophage as an aid to rapid DNA sequencing. J. Mol. Biol. 143:161-178.

18. Earnshaw, W. C., K. F. Sullivan, P. S. Machlin, C. A. Cooke, D. A. Kaiser, T. D. Pollard, N. F. Rothfield, and D. W. Cleveland. 1987. Molecular cloning of CDNA for CENP-B, the major human centromere autoantigen. J. Cell Biol. 104:817-829.

19. Gonzalez, F. J., T. Matsunaga, K. Nagata, U. A. Meyer, D. W. Nebert, J. Pastewka, C. A. Kozak, J. Gillette, H. V. Gelboin, and J. P. Hardwick. 1987. Debrisoquine 4-hydroxylase: Characterization of a new P450 gene subfamily, regulation, chromosomal mapping and molecular analysis of the DA rat polymorphism. DNA. 6:149-161.

20. Gut, J., T. Catin, P. Dayer, T. Kronbach, U. Zanger, and U. A. Meyer. 1986. Debrisoquine/sparteine-type polymorphism of drug oxidation. Purification and characterization of two functionally different human liver cytochrome P-450 isozymes involved in impaired hydroxylation of the prototype substrate bufuralol. J. Biol. Chem. 261:11734-11743.

21. Meier, U. T., and U. A. Meyer. 1987. Genetic polymorphism of human cytochrome P-450 (S)-mephenytoin 4-hydroxylase. Studies with human autoantibodies suggest a functionally altered cytochrome $\mathrm{P}-450$ isozyme as cause of the genetic deficiency. Biochemistry. 26:8466-8474.

22. Idle, J. R., and R. L. Smith. 1979. Polymorphisms of oxidation at carbon centers of drugs and their clinical significance. Drug Metab. Rev. 9:301-317.

23. Schalm, S. W. 1982. Treatment of chronic active hepatitis. Liver. 2:69-76.

24. Zanger, U. M., H.-P. Hauri, J. Loeper, J.-C. Homberg, and U. A. Meyer. 1988. Antibodies against human cytochrome P450db1 in autoimmune hepatitis type II. Proc. Natl. Acad. Sci. USA. 27:82568260.

25. Gueguen, M., M. Meunier-Rotival, O. Bernard, and F. Alvarez. 1988. Anti-liver kidney microsome antibody recognizes a cytochrome P450 from the IID subfamily. J. Exp. Med. 168:801-806. 